\title{
Visualizing Student Behavior and Performance in an Online Course
}

\author{
Michael J. Parker, MD* \\ Harvard Medical School
}

\begin{abstract}
Online learning has been an area of tremendous growth in recent years [1], further accelerated by necessity during the coronavirus pandemic [2]. Without the feedback provided by synchronous sessions, however, instructors may lack ongoing insight into students' progress and performance in fully asynchronous online offerings. Providing greater visibility into students' online learning behavior has several potential benefits: 1) teachers who seek to provide live sessions that depend on knowledge from asynchronous resources will have a better gauge of students' preparation; 2) enhanced understanding of the relationship between student pacing and performance can help teachers and researchers characterize the impact of timely engagement with online course material; 3) providing a means of monitoring the effect of changes in course structure or incentives may guide course designers/planners in continuous improvement; and 4) visualizations that illustrate the relationship between students' course progress and performance have the promise of allowing teachers to take steps early to positively affect students' outcomes. In this visual case study, visualizations are provided for exploration of the relationship between student progress and performance in an online medicinerelated course.
\end{abstract}

Keywords: temporal data; application motivated visualization; education; online learning.

Index Terms: K.3 [Computers and Education]: Computer Uses in Education

\section{Motivation}

How do students move through the problems (assessment questions) in a fully online course over time? In a web-based notebook (see https://observablehq.com/d/1f8bcd443c29b22b), I present an interactive visualization of assessment-taking on a dayby-day basis over a multi-week course. The motivation for this visualization is that, unlike an on-the-ground class where students are sitting in front of a teacher, most, if not all, activity in an online course may be invisible to a course teacher or administrator in the absence of analytics that show students' progress and performance. In addition, there are multiple potential benefits, as outlined in the abstract, to better understanding the relationship between behavior and performance.

Based on personal experience in running online courses with schools around the world, it is also common for teachers to have an expectation that students taking an online course progress through released material in a relatively timely fashion. But is this the norm

\footnotetext{
* michael_parker@hms.harvard.edu
}

or not? Students' behavior in terms of progress and performance is conventionally conveyed as static snapshots in the form of spreadsheets of intermediate grades, which do not lend insight into the time progression of students' engagement with course material.

\section{Visualization Description}

After viewing a brief description of an online course structure (shown visually in notebook Figure 1 and with learner behavior in notebook Figures 2 through 5), users are able to play or scrub through the two interactive diagrams, notebook Figure 6 and notebook Figure 7, which show two synchronized representations of 40 students moving through an online course.

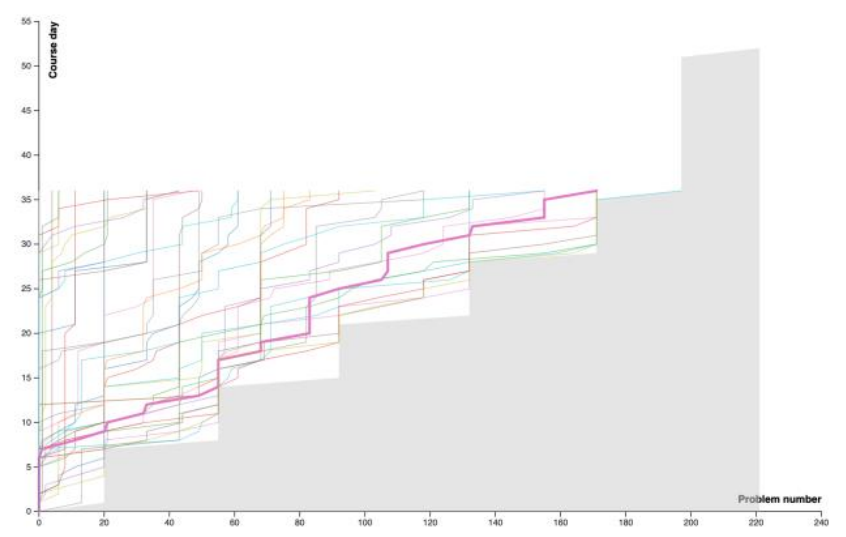

Figure 1: Static image of interactive notebook Figure 6 (Student assessment-taking progress, shown paused at day 36 of the online course

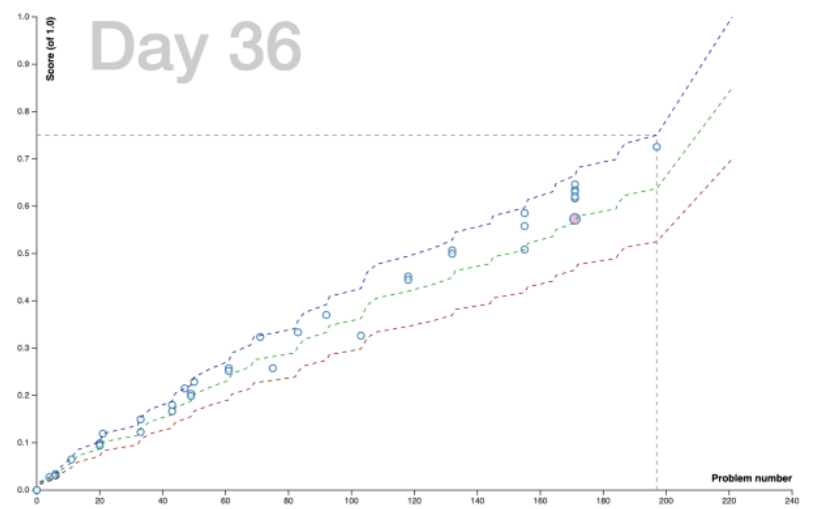

Figure 2: Static image of interactive notebook Figure 7 (Student performance, shown paused at day 36 of the online course

The first shows students' progress (number of assessment questions taken through each course day) as the course progresses and more assessment questions are released. The second diagram is 
synchronized with the first and shows student performance (total score out of 1.0) achieved by each student as they take released assessment questions.

As a user explores the diagrams, they can select one or more students in either diagram to more easily follow the corresponding progress trace or animation in the other diagram. The course day vs problem number interactive plot (notebook Figure 6) allows straightforward identification of students who start late or who demonstrate even or uneven pacing in their assessment-taking behavior, and the synchronized score vs problem number plot (notebook Figure 7) lets the user directly follow the scoring progression or outcomes of those students.

\section{Data Description}

The student score and timing data is derived from an online physiology course run with a medical student cohort. The course platform is an instance of the Open edX learning management system, with data collected in a MySQL database, including scoring and timing on a per learner, per assessment question basis. Course structure, including number of assessment questions and weighting of individual questions and quizzes (groupings of questions), is stored in a MongoDB database. The student performance score metric is derived through a weighted summation of quiz scores, with $75 \%$ weight placed on a student's score in body of the course (196 questions) and 25\% weight placed on the final exam score ( 25 questions). The dashed lines representing $100 \%$, $85 \%$, and $70 \%$ of available points in Figure 7 of the referenced visualization notebook are not straight because quizzes with equal weight may have slightly different numbers of questions and therefore individual questions may differ in point value.

\section{OUtcomes}

Based on using this visualization during and after an online course, it is expected that instructors will be better able to understand students' learning behavior, as well as more intuitively see relationships, for example, between procrastination and performance, and between interventions and changes in students' behavior. Interesting questions to explore include: 1) do students who start later end up scoring worse in the course? 2) do students who are more evenly paced or work through the material over a greater number of days end up scoring better? and 3) can well-timed interventions, facilitated through better understanding of students' behavior, affect learning outcomes?

Ideally, struggling students may also be identified earlier in a course based on correlated views of their progress and performance.

\section{Next Steps}

This visualization will be incorporated into a web-based dashboard driven by real-time or near real-time data from ongoing courses and available to course instructors during the course period. We will measure instructors' usage of this tool, as well as conduct interviews to better assess whether they gain a better understanding of the data and the actions they take based on that knowledge.
Additionally, I plan to analyze the existing data on the more than 20,000 students who have already been through these medicinerelated online courses [3] using visualization approaches more amenable to large datasets as well as machine-learning techniques to gain more generalizable insight into correlation between behavior and performance and to aid in prediction of outcomes. In addition to studying comparisons between individual learners, given the large number of cohorts for which data exists, comparisons between cohorts may yield interesting conclusions.

Visualization challenges that I plan to further address for presentation of data in this format include maintaining clarity and avoiding overplotting for cohorts in which there are larger numbers of students (typical medical school cohorts have between 50 and 200 students, but may sometimes have more than 200), and experimenting with formats in which correlation between the animated plots can be seen without vertical scrolling.

\section{REFERENCES}

[1] Li, C. and Lalani, F, The COVID-19 pandemic has changed education forever. This is how, 2020. Accessed on: 14 June 2021. [Online] Available: https://www.weforum.org/agenda/2020/04/coronaviruseducation-global-covid19-online-digital-learning/

[2] Shah, D., By The Numbers: MOOCs in 2020, 2020. Accessed: 14 June 2021. [Online]. Available: https://www.classcentral.com/report/mooc-stats-2020/

[3] HMX Online Learning, Harvard Medical School. Accessed: 14 August 2021. [Online]. Available: https://onlinelearning.hms.harvard.edu. 